\title{
Chromatic behavior of amplitude and phase compensators
}

\author{
Laurent Pueyo, Michael G. Littman, Jeremy Kasdin, Ruslan Belikov, \\ and Amir Give'on
}

Department of Mechanical and Aerospace Engineering, Princeton University

\begin{abstract}
The achievable contrast level for space-based detection of exo-planets will be limited by the stability of the optics. As a consequence, active amplitude and phase compensation will be needed. High order aberrations can arise from both non linearity and Fresnel propagation, their wavelength dependence is studied here and it is concluded that $\lambda$ independent errors are dominant. The behavior of a Michelson interferometer equipped with two deformable mirrors under chromatic light is then presented, and an achromatization method based on a dispersive element is suggested.
\end{abstract}

\section{Reflectivity errors and Fresnel induced errors}

\subsection{Contrast degradation due to amplitude errors.}

Visible light imaging of exo-planets will require both an instrument whose intrinsic design enables high dynamic range imaging and an extremely stable wavefront. In order to achieve successful planet detection the TPF mission will need a $10^{-10}$ contrast ratio in the zone of the image plane where planets photons are expected to land, called "dark zone". At this extreme level of starlight extinction, not only phase errors have to be corrected but amplitude fluctuations have to be taken into account 1. Maintaining a uniform illumination in spite of the uneven reflectivity of the primary mirror is the main problem justifying the need for an active amplitude compensator, but other amplitude errors can arise as a consequence of Fresnel propagation.

\subsection{Expansion of the pupil plane aberration}

In order to understand the wavelength behavior of Fresnel induced errors we will expand the aberration as a sum of $\lambda^{k}$, with $k \in \mathbb{Z}$. This approach relies on the propagation of spatially periodic aberrations, so we will adopt a Fourier representation for both the amplitude and the phase error. The phase $\phi(x)$ will be decomposed in harmonic series with Fourier coefficients $\left\{\frac{b_{l}}{\lambda}\right\}_{l \in \mathbb{Z}}$, the reflectivity $r(x)$ will be represented by the series $\left\{a_{k}\right\}_{k \in \mathbb{Z}}$. Expanding the phase exponential leads to a more explicit wavelength dependence of the aberration:

$a b b(x)=(1+r(x)) e^{j \phi(x)}=1+a_{k} e^{j 2 \pi \frac{k}{D} x}+j \frac{b_{l}}{\lambda} e^{j 2 \pi \frac{l}{D} x}+a_{k} \frac{b_{l}}{\lambda} e^{j 2 \pi \frac{m(k, l)}{D} x}+\left(\frac{b_{l}}{\lambda}\right)^{2} e^{j 2 \pi \frac{l}{D} x}+\ldots$

The next step is to evaluate the Fresnel integral for each one of these terms.

\subsection{Fresnel propagation of a Fourier component}

We choose not to take into account the $\frac{1}{\lambda z}$ factor in front of the propagation integral, and to consider the case of an infinite aperture. Then, if we isolate a given spatial frequency 
in the first order of the phase expansion, the field in the pupil plane after a distance $z$ is:

$$
E(\xi, z)=\int_{-\infty}^{+\infty}\left(1+j \frac{b_{l}}{\lambda} e^{j 2 \pi \frac{l}{d} x}\right) e^{j \frac{\pi}{\lambda z}(x-\xi)^{2}} d x
$$

The integration of the constant yields a uniform field.The second part has a closed form that can be written as:

$$
E_{2}(\xi)=j \frac{b_{l}}{\lambda} e^{j 2 \pi \frac{l}{d} x} e^{j \pi \frac{l^{2}}{D^{2}} \lambda z}
$$

This result exhibits a coupling between real and imaginary part of the aberration. This Fresnel factor justifies the existence of phase induced amplitude errors and amplitude induced phase errors. In order to generalize this result to a finite aperture we turned ourselves to numerical simulations: the case of a $D=1 \mathrm{~m}$ gaussian apodized pupil, under a $\lambda_{0}=600 \mathrm{~nm}$ incident light was considered, the original aberration was phase only, pure imaginary. As shown on figure 1, after $10 \mathrm{~m}$ the periodic component of the field becomes both real and imaginary: we observe a phase induced amplitude error. After a propagation of a distance $z_{T}=\frac{2 D^{2}}{\lambda l^{2}}$ the initial pupil is retrieved which concurs with the periodicity of the fresnel factor derived above.

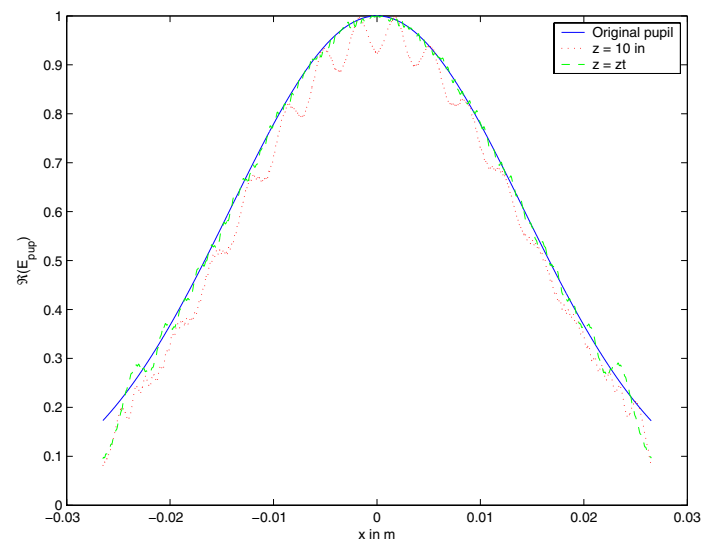

Figure 1. Illustration of phase induced amplitude errors: real part of the near field.

\subsection{Final expansion of the field}

Since $\pi \frac{l^{2}}{D^{2}} \lambda z$ is small, the final step in our expansion of the pupil plane field is to consider a Taylor series of this Fresnel factor. Then, every term in equation (1.1) is multiplied by a polynomial in $\lambda$. The final expression for the field at any distance $z$ in the optical train can then be written as:

$$
E_{z}(\xi)=\sum_{n} c_{n}(\lambda, z) e^{j 2 \pi \frac{n}{D} x}
$$

Table (1) summarizes the contribution the wavelength dependence of the coefficients $c_{n}$ and the contribution of the $a_{k}$ and $b_{k}$ in this final expansion. Note the wavelength independence of the phase induced amplitude term, whose spectral behavior is thus similar to reflectivity errors. Since, to the first order, amplitude errors are wavelength independent, a truly polychromatic amplitude actuator becomes necessary. 
Expansion of the aberration

\begin{tabular}{l|cccc} 
Fresnel Factor & $\mathrm{i} \frac{b}{\lambda}$ & $a$ & $i \frac{a b}{\lambda}$ & $\frac{b^{2}}{\lambda}$ \\
1 & $i \frac{b}{\lambda}$ & $a$ & $\frac{a b}{\lambda}$ & $\frac{b^{2}}{\lambda}$ \\
$i \frac{\pi z k^{2}}{D^{2} \lambda}$ & $\frac{\pi z k^{2}}{D^{2}} b$ & $i \frac{\pi z k^{2} \lambda}{D^{2}} a$ & $\frac{\pi z k^{2}}{D^{2}} a b$ & $\frac{\pi z k^{2}}{D^{2} \lambda^{2}} b^{2}$
\end{tabular}

Table 1. Wavelength dependence of each term in the expansion of a Fresnel Propagated aberration.

\section{Chromatic leakage in a zero path length difference interferometer}

\subsection{Michelson interferometer equipped with two deformable mirror}

In this section we consider a zero path length difference Michelson interferometer used as an amplitude compensator2. Two deformable mirrors provide two degrees of freedom, and using them in an interferometric configuration enables to convert their phase contribution into Path Length Difference and thus into amplitude variations. This can be written as:

$$
\underline{E_{\text {out }}(x, y)}=\frac{E_{\text {in }}(x, y)}{2} \cos \left(\frac{\pi}{\lambda}\left(z_{1}(x, y)-z_{2}(x, y)\right)\right) \exp \left(j \frac{\pi\left(z_{1}(x, y)+z_{2}(x, y)\right.}{\lambda}\right)
$$

Where $E_{i n}$ is the incident electrical field, $E_{\text {out }}$ the outgoing one, $z_{1}$ and $z_{2}$ are the heights of each deformable mirror, and $\lambda$ is the wavelength of the incoming light.

\section{Chromatic leakage of a classical interferometer under polychromatic light}

While phase correction is polychromatic, the amplitude compensation is only perfect for a central $\lambda_{0}$ and all other incident wavelengths will leak and degrade the contrast. As an example, if all the energy of a $\lambda$ independent aberration is contained in one big speckle, a contraint of 10 orders of magnitude in contrast leads to the requirement of $\delta \lambda / \lambda=\frac{1}{20}$, with an amplitude error of $5 \times 10^{-4}$. While the dominant amplitude errors are wavelength independent, the expansion in (1) suggests to evaluate the influence of a zero path length difference interferometer on $1 / \lambda^{k}$ errors. Figure 3 plots $\langle\log (P S F)>$ as a function of the bandwidth for different wavelength dependences of the aberration. For $k=2$ the correction is actually polychromatic. This result can be proved analytically by using a Taylor expansion of $(2.1)$, which is justified since $d(x) / \lambda$ is expected to be small. The cancellation of a $E_{a b b}=E_{0}(1+\epsilon \cos (f x))$ amplitude ripple using an harmonic $\mathrm{PLD}, d(x)=a_{\text {corr }} \cos \left(f_{\text {corr }} x\right)$, leads to solve (3.1) for $a_{\text {corr }}$.

$$
E_{\text {out }}(x)=\left(1+\frac{\epsilon}{\lambda^{2}} \cos (f x)\right)\left(1-\frac{\pi^{2} a_{\text {corr }}^{2}}{2 \lambda^{2}} \cos ^{2}\left(f_{\text {corr }} x\right)\right)
$$

Hence, by choosing $a_{\text {corr }}^{2}=\frac{4 \epsilon}{\pi^{2}(1+\epsilon)}$ and $f_{\text {corr }}=f / 2$, polychromatic correction can be achieved in the case of $1 / \lambda^{2}$ amplitude errors.

\section{Achromatization using a dispersive cell}

The correction of $\lambda$ independent amplitude errors remains the biggest concern since they are the largest source of luminosity non uniformity. In an effort to circumvent any chromatic leakage associated to them, we suggest in this section a concept of polychromatic amplitude control.

\subsection{Insertion of a dispersive gaseous cell}

Figure 4(b) illustrates the insertion of a gas cell in a one leg of a Michelson interferometer. The ides of tuning the a path length difference using dispersion and pressure changes has 


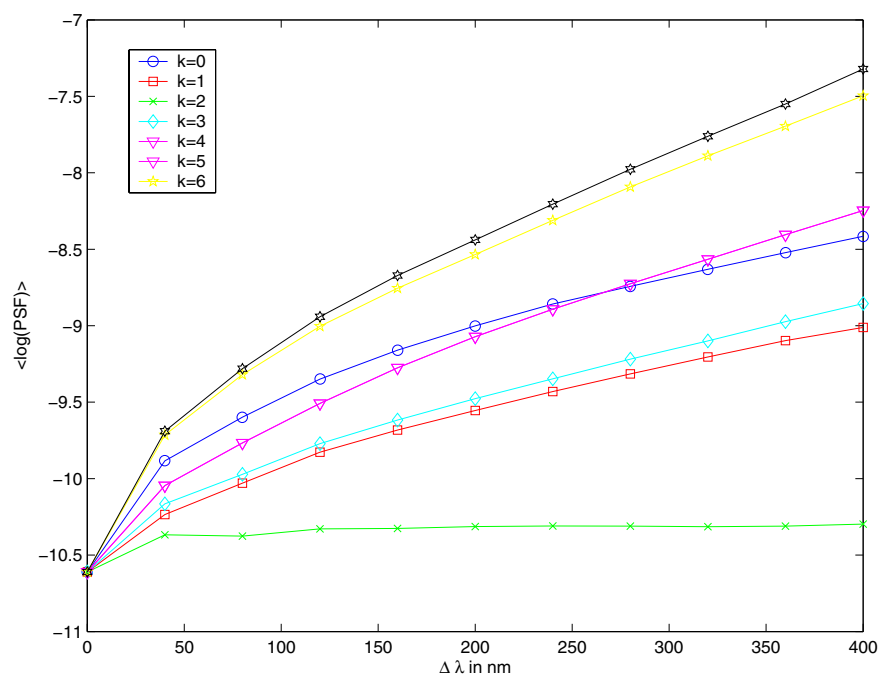

Figure 2. Chromatic leakage for several $1 / \lambda^{k}$ dependence.

been used since the 50's, when Jacquinot 3 tuned a Fabry Perot spectroscope by enclosing it in a pressurized chamber. For our application we consider a cell with a gas having the linear dispersion $n(\lambda)=1+\alpha(P) \lambda$, where $P$ is the pressure of the gas within the cell. Figure 3(a) illustrates the chromatic behavior of a classical Michelson interferometer, for which the response to a given Path Length Difference varies with wavelength. As stated previously, chromatic leakage is minimized if the amplitude compensator operates around Zero Path Length Difference. Using a dispersive gas enables to operate at constant ZPLD and introduces what is the equivalent of a polychromatic phase shift, as seen on figure 3(b). The necessary degree of freedom to control the amplitude, or to adjust the height of the intersection with the y axis, would come from a pressure adjustment in the gas cell.

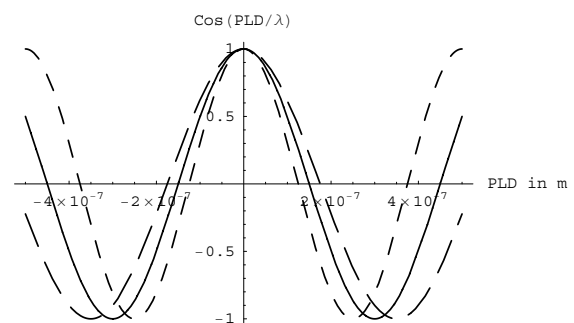

(a) Profile of the amplitude response of classical Michelson interferometer

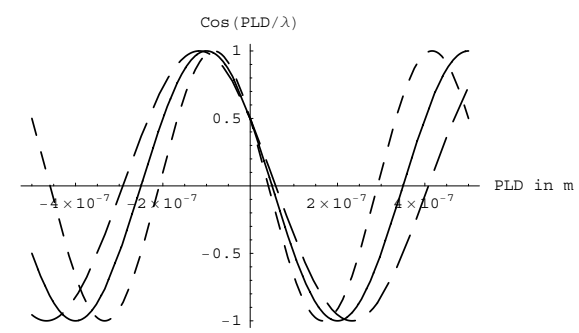

(b) Profile of the amplitude response of a Michelson interferometer with a gas cell inserted in one leg

Figure 3. Comparison of the response of the interferometer for three wavlength: $500 \mathrm{~nm}$, short dashes, $600 \mathrm{~nm}$, full line, $700 \mathrm{~nm}$, long dashes.

A pixelated polychromatic amplitude control could thus be obtained by using two flat mirrors and stacking together several cells with independently controlled pressure. We are 
aware that such a system requires adressing technical issues that go far beyond the scope of this paper. Our objective here is merely to introduce a novel idea for polychromatic amplitude control, a critical component of the TPF mission, leaving aside technological feasibility for further studies.

\subsection{Proof of concept: linear dispersion of nitrogen}

The fundamental assumption underlying the achromatization is that the gas is linearly dispersive. This feature is confirmed experimentally for Nitrogen by Peck and Khanna 4 . Figure 4(a) has been drawn for $P_{0}=1 \mathrm{~atm}$ and $T_{0}=288 \mathrm{~K}$, the dotted line is the actual dispersion profile, the influence of the error introduced by the linearization remains under the critical value of the $10^{-5}$ requirement for the electrical field magnitude.

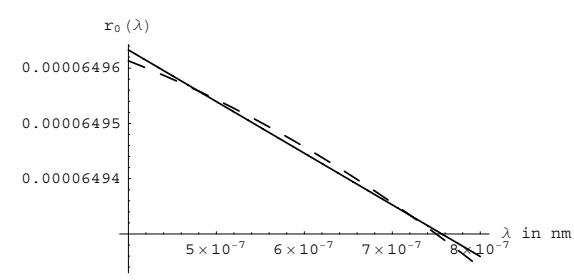

(a) $r_{0}(\lambda)$ in the visible, note the quasi linearity

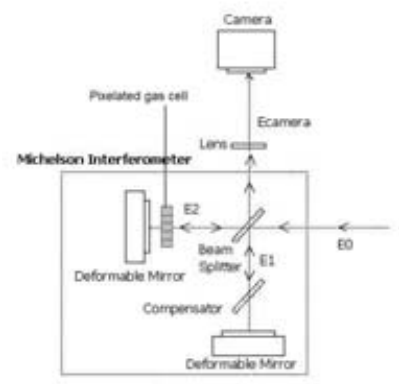

(b) Polychromatic Michelson interferometer with a gas cell

Figure 4. Concept of a dispersive element in an interferometer, and dispersion of Nitrogen in the visible.

\section{Conclusion}

The necessity to compensate for amplitude errors in coronographs dedicated to exoplanet detection comes from tight contrast requirements and Fresnel propagation of aberration has thus to be taken into account. We introduced a systematic approachto studying the chromatic effects of aberrations that is the first step to an eventual polychromatic correction. The interesting behavior of an interferometer a broadband corrector for $1 / \lambda^{2}$ aberrations is proved, and chromatic leakage is quantified for other wavelength dependencies. Finally we proposed a concept to correct for $\lambda$ independent amplitude errors and showed a short proof of its validity based on a literature review. Our next step is to carry out this idea experimentally.

\section{References}

Brown, R.A. \& Burrows, C.J 1990, Icarus 87, 484

Littman, M.G., Carr, M., Leighton, J., Burke, E., Spergel, D.N., \& Kasdin, N.J. (2002), Proc SPIE 4860, 182

Jacquinot, P. 1954, JOSA 44, 761

Peck, E. \& Khanna, B.N. (1966) JOSA 56, 1059 

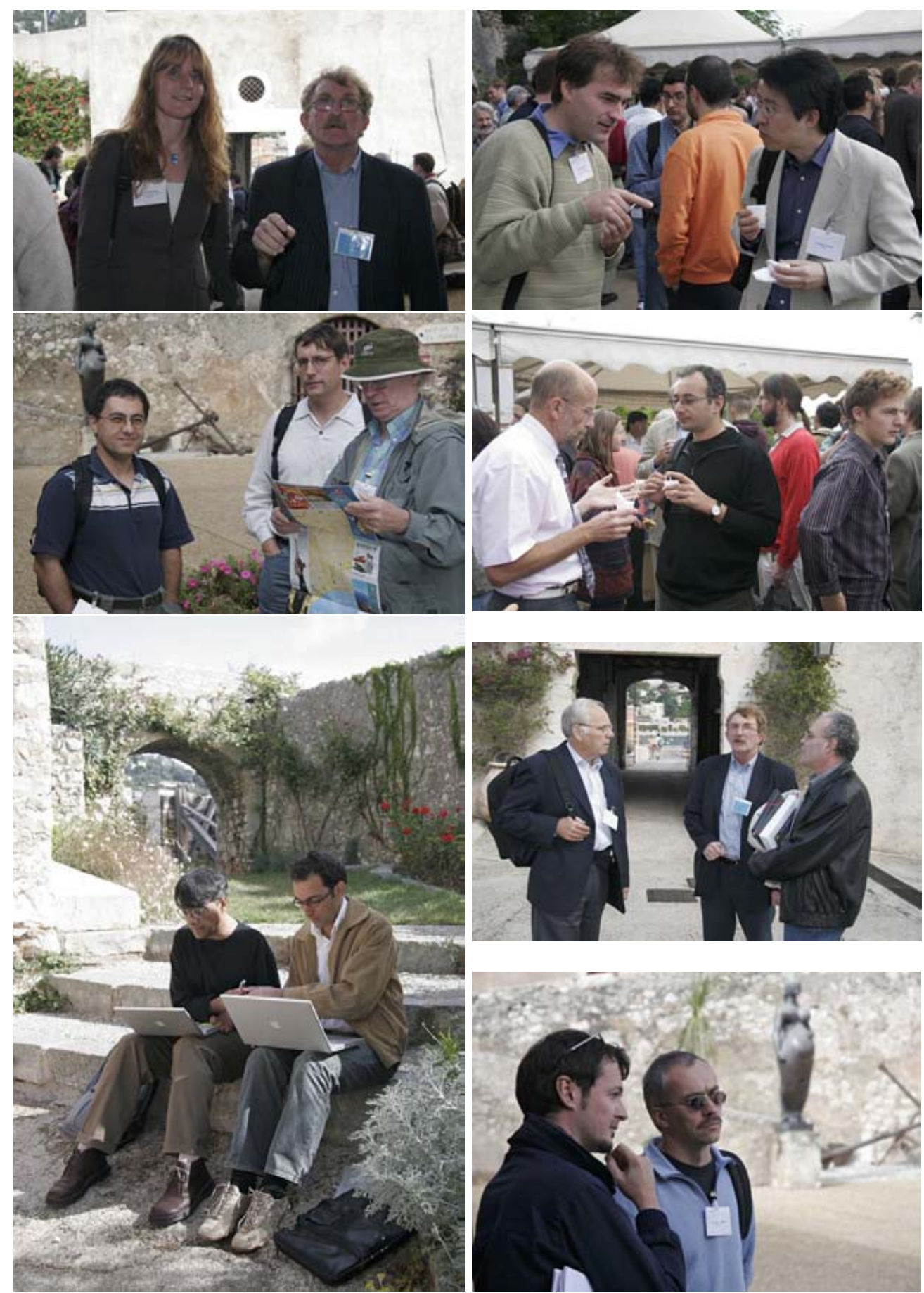

All photographs: Laurent Thareau [1.thareau@free.fr]. 